\title{
Novo Qualis: Impacto na Avaliação da Produção Intelectual dos Pesquisadores em Administração
}

\author{
New Qualis: Impact in the Assessment of the Intellectual \\ Production of Management Researchers
}

Tatiane Baseggio Crespi

Mestranda do Programa de Pós Graduação em Administração da Universidade Nove de Julho. Passo Fundo, Rio Grande do Sul. Brasil.E-mail: tatianebaseggiocrespi@gmail.com

\section{Taísa Scariot Preusler}

Mestranda do Programa de Pós Graduação em Administração da Universidade Nove de Julho. Passo Fundo, Rio Grande do Sul. Brasil.E-mail: taisa.s@hotmail.com

\section{Nestor Alejandro Luna}

Mestrando do Programa de Pós Graduação em Administração da Faculdade Meridional -IMED. Passo Fundo, Rio Grande do Sul. Brasil.E-mail: alelunabr@hotmail.com

\section{Manuel Portugal Ferreira}

Professor de Estratégia no Programa de Pós-Graduação em Administração, Uninove e no Departamento de Gestão, ESTG, Portugal. Leiria, Portugal.E-mail: manuel.portugal.ferreira@gmail.com

\section{Resumo}

Neste estudo analisa-se a avaliação da produção intelectual - artigos publicados em periódicos científicos - dos professores pesquisadores em Administração usando indicadores quantitativos. Em 2015, a CAPES definiu os critérios de um novo Qualis para o quadriênio 2013-2016 que teve efeitos retroativos a 2013, os quais foram alvo de contestação. Com dados coletados dos curriculos Lattes de uma amostra de 97 pesquisadores em Administração e 325 artigos, observa-se o impacto do novo Qualis na pontuação dos pesquisadores. Os resultados mostram que a aplicação do novo Qualis resultou em grande quebra na pontuação científica dos pesquisadores, com eventuais reflexos nos conceitos dos Programas de Pós-graduação em Administração (PPGAs). Este estudo contribui para o debate sobre a avaliação docente indexada a sistemas que ranqueiam periódicos, o impacto específico do Qualis e a mudança nos critérios Qualis na academia brasileira de Administração.

Palavras-chave: Qualis. Indexadores. Produção intelectual. CAPES.

\section{Abstract}

In this study we analyze the assessment of intellectual production - articles published in scientific journals - by management researchers using quantitative indicators. In 2015 CAPES defined the criteria for a new Qualis for the quadrennium 2013-2016 with retroactive effects to 2013, that were highly contested. With data collected from the curriculum Lattes of a sample of 97 management researchers and 325 articles, we examined the impact of the new Qualis on the researchers' evaluation. Results show that the application of the new Qualis led to a significant loss in the researchers' scientific scores, with possible reflections on the evaluation of the doctoral programs. This study contributes to the debate on the evaluation of scholars indexed to systems that rank journals, the specific impact of Qualis, and the change in the Qualis criteria in the Brazilian academy of Management.

Keywords: Qualis. Indexes. Intellectual production. CAPES. 


\section{INTRODUÇÃO}

A publicação científica - ou publicação de artigos científicos em periódicos com revisão pelos pares - é uma das métricas fundamentais na avaliação de professores pesquisadores, programas (mais notavelmente os de stricto sensu) e universidades (STAHL; LEAP; WEI, 1988; MILLER; TAYLOR; BEDEIAN, 2011; FERREIRA, 2015). Esta é uma realidade transversal a muitos sistemas nacionais, dos Estados Unidos, aos países Europeus e mesmo ao Brasil (GAZZOLA, 2008; MACCARI et al., 2008; MACCARI et al., 2009; MACCARI et al., 2014). No Brasil, especificamente, é a Coordenação de Aperfeiçoamento de Pessoal de Nível Superior (CAPES) que cumpre a responsabilidade de definir os critérios de avaliação, inclusive de avaliação dos Programas de Pós-graduação (PPGs) stricto sensu, $e$ a divulgação da produção científica (MACCARI et al., 2008; MACCARI et al., 2009; NASCIMENTO, 2010; CAPES, 2015a).

A avaliação da produção científica dos pequisadores é, usualmente, feita usando indicadores quantitativos relacionados com os periódicos em que publicam e o número de citações aos artigos nesses periódicos - designado, comumente, por fator de impacto. Atualmente, há três fontes de dados mais usadas de citações: Web of Science (WoS), Scopus e Google Scholar (BAKKALBASI et al., 2006). No Brasil, a CAPES desenvolveu um sistema próprio de ranqueamento de periódicos designado por Qualis. O Qualis classifica os periódicos em diferentes estratos segundo diversos critérios, incluindo dados de outros indexadores, como o fator de impacto do Journal Citation Report (JCR), Scopus e SciELO. O Qualis como sistema, é revisado periodicamente e foi alvo de atualização e modificação em 2015, tendo efeitos retroativos. A avaliação da produção científica impacta diretamente os pesquisadores, mas também as universidades e, em especial, os PPGs, em cuja avaliação há uma ponderação significativa da produção intelectual (incluindo a publicação de artigos).

Nesse estudo, analisou-se os principais sistemas de classificação de periódicos e focou-se especificamente no Qualis brasileiro. Analisou-se a evolução das alterações do Qualis, os indexadores que o Qualis utiliza - JCR, Scopus e Scielo - e a relação estabelecida pelo Qualis e a avaliação dos PPGAs. A abordagem empírica consistiu na análise com natureza descritiva do impacto da alteração do novo Qualis na pontuação dos professores pesquisadores. Esta análise é necessária dado que em 2015 a CAPES liberou os novos critérios de avaliação dos periódicos que determina a pontuação dos pesquisadores. Face às críticas que este novo Qualis para o Quadriênio 2013-2016 gerou pelo impacto na contabilização da produção científica realizada em 2013 e 2014, analisou-se o impacto da alteração no Qualis na avaliação da produção intelectual dos pesquisadores. Para esta análise foram coletados dados secundários dos currículos Lattes de uma amostra aleatória de pesquisadores afiliados a programas stricto sensu, do Portal Sucupira e dos cadernos de indicadores da CAPES.

Este estudo tem, assim, duas contribuições primordiais. A primeira, para a melhor compreensão do impacto dos sistemas quantitativos de avaliação do desempenho científico dos professores pesquisadores em Administração. Foi possível, assim, melhor entender a relação entre as publicações em periódicos classificados nos diferentes estratos do Qualis e a avaliação dos pesquisadores e dos PPGAs, no que se refere à avaliação da produção intelectual. A segunda contribuição é de cariz mais prático, consistindo na mensuração do impacto real das alterações introduzidas no Qualis, em 2015, que retroagem, na pontuação dos professores pesquisadores. Diante das mudanças introduzidas é relevante entender o impacto que as alterações no Qualis tiveram na avaliação do desempenho científico dos pesquisadores e que poderão ter o seu reflexo na avaliação CAPES dos PPGAs.

\section{Revisão da Literatura}

As revistas científicas começaram a ser publicadas no século XVII e desde então têm sido o veículo fundamental na comunicação científica (STUMPF, 1996). As revistas científicas são um dos principais canais de divulgação dos resultados da investigação, institucionalizada na maioria das áreas do conhecimento. Seu reconhecimento depende em grande medida da sua qualidade e visibilidade (MIGUEL, 2011).

O grande crescimento de periódicos e do número de artigos publicados torna ao pesquisador mais dificil saber o que ler, e quais os artigos relevantes para a 
pesquisa ou mesmo o ensino (FERREIRA, 2015). É comum a assunção que os periódicos de mais alto status são mais lidos e que seus artigos têm maior impacto. A qualificação dos periódicos é usualmente mensurada com o fator de impacto (GARFIELD, 1972, 2006) publicado anualmente pela Thomson Reuters, ou as métricas alternativas da Scopus (pela editora Elsevier), ou da ScieLO. Em essência, independentemente da origem, os indicadores do impacto de periódicos usam as citações aos artigos que publicam para quantificar a sua influência e para inferir qualidade relativa.

O desempenho dos pesquisadores no quesito da produção científica tem vindo a ser aferido pelo impacto dos periódicos em que publicam os seus trabalhos (STAHL et al., 1988; VELASCO et al., 2012; FERREIRA, 2015). O prestígio dos autores e a qualidade percebida das suas publicações estão associados com a qualidade (ou ranqueamento) dos periódicos. Esta avaliação reflete na contratação, promoção na carreira, financiamento, etc. (FIATES; SERRA; MARTINS, 2014; OLIVEIRA; SANTIN; VANS, 2015; TRUEGER et al., 2015).

Muito embora a sua popularização (COELHO et al., 2003), a utilização de fatores de impacto tem sido amplamente criticada (ver SEGLEN, 1997; HECHT et al., 1998; ADAM, 2002; COELHO et al., 2003; TREIN; RODRIGUES, 2011; SHUGAN, 2013). Algumas das críticas notadas incluem a lentidão para acumular citações, enquanto os requisitos das universidades são mais imediatistas, o elitismo (não considera bons artigos em periódicos menos reputados), a possibilidade de auto-citações ou citações de amigos ou grupos e o fato de apenas contemplar publicações em inglês (MENEGHINI; PACKER, 2007). Talvez a maior crítica seja que as citações medem apenas o impacto quantitativo e não a real influência intelectual, mais qualitativa, dos artigos (VELASCO et al., 2012).

Hirsch (2005) propôs o índice H (ou h-index) para avaliar o impacto dos pesquisadores, em vez dos periódicos e, assim, ultrapassar uma das críticas aos fatores de impacto. Em essência, o índice $\mathrm{H}$ combina o número de artigos do pesquisador com uma métrica de impacto (número de citações aos artigos) para mensurar a contribuição do pesquisador (BAR-LLAN, 2008). Outros autores avançaram formas de medir o impacto não de periódicos ou de pesquisadores, mas de cada artigo, que designam por altmetrics (TRUEGER et al., 2015), baseado também na divulgação nas mí- dias sociais e não apenas em outros artigos científicos tradicionais. Uma iniciativa interessante é o software de Anne-Wil Harzing em 'publish or perish' (ver em http:// www.harzing.com/resources/publish-or-perish) que coleta e analisa, segundo várias métricas, as citações acadêmicas de artigos de um pesquisador.

Em todo o caso, a avaliação da produção científica é notadamente um processo complexo, difícil e sem uma solução prática adequada. Idealmente as publicações seriam analisadas por peritos na área que avaliariam a qualidade segundo regras previamente acordadas. Na prática, no entanto, utilizam-se medidas secundárias como o prestígio do periódico, a reputação dos autores e das organizações de origem e as contagens de publicações realizadas (SEGLEN, 1997).

As questões relativas à avaliação da produção científica são tão mais relevantes na academia quanto se vive num período de 'publicar ou perecer' ('publish or perish' - ver Harzing, 2013) que Rosa (2008) notou ser o reflexo da pressão institucional sobre as publicações. Tourinho e Palha (2014) colocaram que os critérios impostos para a produção induzem à alienação. Por outro lado, Gaspar e Campos (2015) consideram que a valorização da produção científica dos PPGAs motiva a busca do aumento qualitativo e quantitativo das publicações científicas, melhorando os resultados nas avaliações trienais destes programas.

\subsection{Avaliação pela CAPES e a Importância da Produção Científica}

No Brasil há um sistema próprio de mensuração da qualidade dos periódicos, que é instituido pela CAPES, designado por Qualis. Fundada em 1951, a CAPES tem atuado na consolidação dos cursos stricto sensu no Brasil. Em 1981, a CAPES teve seu papel fortalecido ao obter o reconhecimento como órgão responsável pela elaboração do Plano Nacional de Pós-graduação stricto sensu (CAPES, 2015a). Com esta atribuição, vem buscando ampliar e solidificar os cursos de mestrado e doutorado no Brasil e contribuir para o desenvolvimento da pesquisa científica e tecnológica. Dentre as suas incumbências, duas destacam-se por sua importância e discussão no meio acadêmico (ver TOURINHO; PALHA, 2014): a avaliação dos PPGs stricto sensu e o acesso e divulgação da produção científica. Ambas cruciais para o êxito do Sistema Nacional de Pós-graduação no país (CAPES, 2015a). 
Um dos vetores de atuação da CAPES é a avaliação dos PPGs. Em 1998, a CAPES implementou um sistema para padronizar os critérios de qualidade para notas, produção científica e formação de alunos, como forma de aperfeiçoamento do desempenho dos programas. Para Sampaio (2006), essa mudança foi um fato importante para a avaliação dos programas. E, segundo Maccari et al. (2008) e Gazzola (2008), este sistema de avaliação dos PPGs é um dos mais eficazes e modernos do mundo. Embora em concordância com os aspectos positivos, Kuenzer e Moraes (2005) apontaram a intensificação produtivista como fator negativo, onde a avaliação quantitativa se destaca em detrimento da qualitativa e onde a quantidade é meta. Alcadipani (2011) corroborou essa ideia, pois entende que as publicações resultam de pesquisas complexas que demandam tempo para serem desenvolvidas. No intuito de aumentar a quantidade para atingir metas, pode prejudicar a qualidade das produções.

O sistema de avaliação dos PPGs não é estático, sendo revisado periodicamente pela CAPES para seu aprimoramento e busca de excelência (MACCARI et al., 2008). Maccari et al. (2009) relataram, embora com algumas ressalvas, que há concordância dos coordenadores dos PPGAs com a classificação adotada pelo sistema de avaliação da CAPES. Sendo a sua estratificação, um fator de distinção de qualidade, orientado para a melhoria evolutiva. Critérios bem definidos e conhecidos influenciam o desenvolvimento dos PPGs. Por exemplo, na área de Administração, o crescimento no número de programas de mestrado $e$ doutorado desde 1998 foi acompanhado pela CAPES para garantir qualidade (MACCARI et al., 2008).

O sistema de avaliação dos PPGs iniciou com o intuito desenvolver a qualidade da educação (NASCIMENTO, 2010). Os PPGs passaram a ser avaliados de forma mais ordenada, no final dos anos 80 e início dos anos 90. No biênio de 1996/1997, um novo modelo de avaliação foi instituído. Até esse período a avaliação era feita com visitas aos programas seguindo critérios definidos em cada área (SOUSA; MACEDO, 2009). Posteriormente, a avaliação passou a ter maior foco na produção bibliográfica (KUENZER; MORAES, 2005).

Sistemas da avaliação de PPGAs vêm sendo utilizados em vários países. A institucionalização do sistema de avaliação dos programas de pós-graduação brasileiros tornou-se uma ferramenta importante para geração de melhorias nos sistemas educacionais, sendo considerado eficiente e moderno (MACCARI et al., 2014). Esse sistema de avaliação tem suas métricas periodicamente reexaminadas e atualizadas (Falaster, Ferreira e Serra, 2016). A classificação Qualis de periódicos é um dos elementos que compõe o sistema de avaliação dos PPGA (FALASTER; FERREIRA; SERRA, 2016), mas também os restantes elementos e critérios de avaliação dos PPGs são alvo de ajustamentos contínuos (FERREIRA, 2015).

Assim, a avaliação pela CAPES conduz os PPGs e os pesquisadores a buscar por publicações em periódicos classificados em estratos Qualis superiores, com maior impacto na pontuação dos pesquisadores e na avaliação dos programas. Neste sentido, despontam, também, estudos que visam compreender todo o processo de pesquisa, publicação e de rejeição de artigos científicos (FERREIRA; FALASTER, 2016). A avaliação CAPES e as pressões para publicar tornam que seja fundamental clarificar os critérios de avaliação, inclusive os de avaliação da produção científica que são orientadores dos esforços dos pesquisadores.

A atual avaliação dos PPGs aplicada pela CAPES é baseada em cinco quesitos: proposta do programa, corpo docente, corpo discente, inserção social e produção intelectual. Os relatórios de avaliação periódica da CAPES explicitam estes quesitos, pelo que uma descrição exaustiva é aqui desnecessária. De forma sumária, o primeiro quesito analisa o programa quanto à coerência, consistência e áreas de concentração, desenvolvimento futuro, e infraestrutura para ensino e pesquisa. O segundo prende-se com a avaliação do perfil do corpo docente, considerando aspectos como titulação, experiência, adequação à proposta do programa, a adequação dos docentes permanentes às atividades de pesquisa $e$ formação, envolvimento na graduação, e outras atividades. O terceiro quesito foca o corpo discente, analisando o número de teses e dissertações defendidas, rácio docentes-discentes, qualidade das teses e dissertações, da produção dos discentes, da eficiência do programa na formação de mestres e doutores, entre outros. O quarto quesito é sobre a avaliação da inserção e impacto regional e/ou nacional do PPG, incluindo a cooperação com outros PPGs e centros de pesquisa (CAPES, 2013a).

O nosso foco relaciona-se com o quinto quesito, que analisa a mensuração da produção intelectual, a 
qual é ponderada em 35\% na avaliação dos PPGAs. A Tabela 1 indica as métricas utilizadas para avaliação da produção intelectual. Integram este componente: publicações qualificadas do programa por docente permanente $(50 \%)$, distribuição de publicações qualificadas em relação ao corpo docente permanente do

Tabela 1: Métricas utilizadas para avaliação da produção intelectual

\begin{tabular}{|c|c|c|c|}
\hline \multicolumn{2}{|c|}{ PuBlicAÇÕES QUALIFICADAS DO PROGRAMA POR DOCENTE PERMANENTE } & \multicolumn{2}{|c|}{$\begin{array}{l}\text { DisTRIBUIÇão DE PUBLICAÇõES QUALIFICADAS EM RELAÇÃo AO } \\
\text { CORPO DOCENTE PERMANENTE DO PROGRAMA (MÉTRICA 1) }\end{array}$} \\
\hline Conceito & Pontuação média anual por docente permanente & Conceito & $\begin{array}{c}\% \text { do NDP que alcançou pelo menos } \\
150 \text { pontos no triênio }(\%)\end{array}$ \\
\hline MB & $>$ ou $=$ a 50 & MB & $>$ ou $=$ a 80 \\
\hline B & $>$ ou $=$ a 35, mas $<$ que 50 & B & $>$ ou $=$ a 70, mas $<$ que 80 \\
\hline $\mathrm{R}$ & $>$ ou $=$ a 30, mas $<$ que 35 & $\mathrm{R}$ & $>$ ou $=$ a 50, mas $<$ que 70 \\
\hline F & $>$ ou $=$ a 12, mas $<$ que 20 & $\mathrm{~F}$ & $>$ ou $=$ a 20, mas $<$ que 50 \\
\hline $\mathrm{D}$ & $<$ que 12 & $\mathrm{D}$ & $<$ que 20 \\
\hline \multicolumn{2}{|c|}{$\begin{array}{l}\text { DisTRIBUIÇÃO DE PUBLICAÇÕES QUALIFICADAS EM RELAÇÃO AO CORPO } \\
\text { DOCENTE PERMANENTE DO PROGRAMA (MÉTRICA 2) }\end{array}$} & \multicolumn{2}{|c|}{ \% DO NDP COM 10 PRODUTOS/ATIVIDADES NO TRIÊNIO } \\
\hline Conceito & Produção bibliográfica qualificada & Conceito & $\%$ do NDP com 10 produtos/atividades no triênio \\
\hline MB & $80 \%$ ou mais do NDP com mais de 200 pontos & MB & $>$ ou $=$ a 50 \\
\hline B & $80 \%$ ou mais do NDP com mais 120 até 200 pontos & B & $>$ ou $=$ a 40, mas $<$ que 50 \\
\hline $\mathrm{R}$ & $80 \%$ ou mais do NDP com mais de 80 até 120 pontos & $\mathrm{R}$ & $>$ ou $=$ a 30, mas $<$ que 40 \\
\hline $\mathrm{F}$ & $80 \%$ ou mais do NDP com mais de 40 até 80 pontos & $\mathrm{F}$ & $>$ ou $=$ a 20, mas $<$ que 30 \\
\hline $\mathrm{D}$ & $80 \%$ ou mais do NDP com 40 pontos ou menos & $\mathrm{D}$ & $<$ que 20 \\
\hline
\end{tabular}

Nota: NDP - Número de Docentes Permanentes. MB - Muito bom. B - Bom. R - Regular. F - Fraco. D - Deficiente.

Fonte: Elaborado pelos autores deste artigo baseado no relatório da CAPES (2013a)

Importa notar que está em debate eventual alteração nas regras da CAPES e que se estima que as mudanças possam contemplar avaliações qualitativas das publicações científicas. No entanto, ao momento, ainda estão em vigor às normas de 2013, pelo que cabe sobre estas uma breve descrição. As publicações qualificadas no programa são avaliadas utilizando-se a pontuação média obtida pela totalidade dos docentes permanentes com base na sua produção intelectual publicada em artigos, livros e capítulos. Para isto, a pontuação acumulada pelo núcleo de docentes permanentes do programa é dividida pelo número de docentes permanentes atuantes no programa no respectivo ano e calculada a média ponderada para avaliação final do triênio (CAPES, 2013a).

Para pontuação dos artigos publicados são utilizados os seguintes critérios, conforme classificação Qualis
Programa (35\%) e produção técnica, patentes e outras produções consideradas relevantes (15\%) (CAPES, 2013a). Ou seja, uma ponderação que incide primordialmente nas publicações de artigos científicos em periódicos qualificados. 
alcançado por $80 \%$ do corpo permanente do programa no respectivo triênio, considerada a métrica 2 . A média ponderada entre estas duas métricas origina o conceito final e é calculada da seguinte forma: (Conceito da Métrica 1 x 2 + Conceito da Métrica 2 x 1)/3. Produção técnica, patentes e outras produções consideradas relevantes, analisam a produção tecnológica e técnica de cada programa, tendo como base a porcentagem do número de docentes permanentes (NDP) com 10 produtos ou atividades no triênio (CAPES, 2013a).

\subsection{Qualis: Evolução e Estado Atual}

Para avaliar o nível da produção científica, a CAPES desenvolveu em 1998, um sistema próprio que é o Qualis. Os procedimentos ou critérios Qualis aferem a qualidade da produção científica por meio da classificação dos periódicos em que os artigos são publicados (NASCIMENTO, 2010; NASCIMENTO; BEUREN, 2011; FERREIRA, 2015; OLIVEIRA et al., 2015). São avaliados periódicos em que pesquisadores brasileiros afiliados em PPGs brasileiros tenham publicado (PACKER, 2011). A classificação dos periódicos pelo Qualis influencia a avaliação dos próprios pesquisadores e, em consequência, dos PPGAs, como visto anteriormente.

No triênio 1998-2000 iniciou-se o planejamento de uma proposta de indicadores para a avaliação da qualidade da produção científica. E, é no triênio de 2001-2003 que a proposta do Qualis foi efetivada, objetivando qualificar periódicos, livros e anais de congressos. Nos anos seguintes, a avaliação dos periódicos foi a que mais evoluiu. Entre 2001 e 2006, os periódicos eram classificados no Qualis em nove estratos, segundo o critério de circulação: internacional, nacional e local, em três níveis, A, B e C e incluía apenas periódicos nacionais. Os critérios contemplavam a normalização, publicação, circulação, conteúdo, autoria e gestão editorial. Era requisitada uma ficha de impacto para verificar quais teriam padrão internacional. O Qualis foi revisado para o triênio 2007-2009. Entre as várias alterações, os estratos passaram a ser estipulados em A1, A2, B1, B2, B3, B4, B5 e C. Indicadores foram estabelecidos para cada um dos estratos, englobando normalização, autoria e conteúdo, gestão editorial e indexação (SOUSA; MACEDO, 2009).

Para o triênio 2010 a 2012, o Qualis foi definido com novos critérios (Tabela 2). E, em 2015, mais alterações no Qualis, que foi definido para o quadriênio 2013 a 2016, portanto, com retroatividade as publicações realizadas em 2013 e 2014. A Tabela 2 expõe os critérios de classificação e permite comparar com o Qualis para 2010-12. A discriminação estabelecida em 2015 coloca maior ênfase no fator de impacto (seja o JCR ou o índice H da Scopus e, em menor grau o SciELO) e na relevância dos periódicos. Mantiveram-se as quotas na distribuição dos periódicos por estratos que devem respeitar as seguintes proporções: $\mathrm{A} 1<\mathrm{A} 2$, $\mathrm{A} 1+\mathrm{A} 2$ no máximo $25 \%$ dos periódicos na base da área, $\mathrm{A} 1$ + A2 + B1 no máximo $50 \%$ dos periódicos na base da área. Periódicos nos estratos B2 à B5 devem representar pelo menos $50 \%$ dos periódicos da área. A imposição de uma distribuição também é alvo de crítica por limitar a classificação, especialmente em estratos superiores (ver ROCHA-E-SILVA, 2009).

Houve exceções à aplicação dos critérios, sendo que alguns periódicos brasileiros em Administração foram classificados num estrato superior do que seriam classificados pelas normas, por serem considerados de importância expressiva. Alguns exemplos incluem o Cadernos EBAPE, RAC, RAE, RBGN, Organizações \& Sociedade (CAPES, 2015b). 
Tabela 2: Comparativo: Qualis para 2010-12 e 2013-16

\begin{tabular}{|c|c|c|}
\hline Estrato & $\begin{array}{l}\text { Critérios para classificação dos periódicos } \\
\text { Qualis 2010-12 }\end{array}$ & $\begin{array}{l}\text { Critérios para classificação dos } \\
\text { periódicos Qualis 2013-16 }\end{array}$ \\
\hline $\mathrm{A} 1$ & $\begin{array}{l}\text { Índice H da Base Scopus (H-Scopus) > } 20 \text { ou; Fator } \\
\text { de Impacto no Journal of Citation Reports (JCR) > } \\
1,0, \text { o que for mais favorável ao periódico. }\end{array}$ & $\begin{array}{l}\text { ISSN; ter no mínimo } 2 \text { edições/ano; JCR }>1,4 \\
(67 \%) ; \text { H-Scopus }>24(75 \%) \text {. Periódicos nos } \\
\text { limites acima, mas não listados como da área, } \\
\text { segundo as bases de cálculo de Fator de Impacto } \\
\text { (FI), foram classificados no estrato A2. }\end{array}$ \\
\hline A2 & $\begin{array}{l}4<\mathrm{H}-S c o p u s \leq 20 \text { ou; } 0,2<\mathrm{JCR} \leq 1,0 \text {, o } \\
\text { que for mais favorável ao periódico. }\end{array}$ & $\begin{array}{l}\text { ISSN; Ter no mínimo } 2 \text { edições/ano; } 1,4>=\text { JCR }> \\
0,7(33 \%) ; 24>=\text { H-Scopus }>9(50 \%) \text {. Periódicos nos } \\
\text { limites acima, mas não listados como da área, segundo as } \\
\text { bases de cálculo de FI, foram classificados no estrato B1. }\end{array}$ \\
\hline B1 & 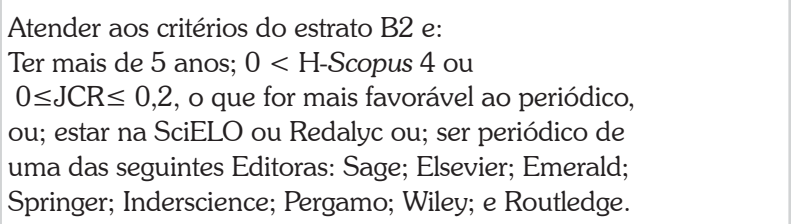 & $\begin{array}{l}\text { ISSN; ter no mínimo } 2 \text { edições/ano; SciELO com } \\
\text { FI }>0,01 \text { e ser da área pelo critério da base, ou } \\
0,7>=\mathrm{JCR}>0 ; 9>=\mathrm{H}-\text { Scopus }>0 \text {. Periódicos } \\
\text { nos limites acima, mas não listados como da } \\
\text { área, segundo as bases de cálculo de Fator de } \\
\text { Impacto, foram classificados no estrato B2. }\end{array}$ \\
\hline $\mathrm{B} 2$ & $\begin{array}{l}\text { Atender aos critérios do estrato B3 e: } \\
\text { Informar sobre os trâmites de aprovação; apresentar a legenda } \\
\text { bibliográfica da revista em cada artigo; ter conselho diversificado; } \\
\text { editor chefe não é autor; informação sobre processo de } \\
\text { avaliação; ter mais de três anos; e ter pelo menos } 1 \text { Indexador } \\
\text { (SCOPUS, EBSCO, DOAJ, GALE, CLASE, HAPI, ICAP, IBSS) }\end{array}$ & $\begin{array}{l}\text { ISSN; ter no mínimo } 2 \text { edições/ano; estar no Redalyc } \\
\text { ou ser editado por Editoras descritas no documento } \\
\text { da área; ou FI- SciELO }<0,01 \text { ou; FI- SciELO }> \\
0,01 \text {, mas de outra área pelo critério da base. }\end{array}$ \\
\hline B3 & $\begin{array}{l}\text { Atender aos critérios do estrato B4 e: } \\
\text { Ter Missão/foco; informar o nome e afiliação do editor; } \\
\text { informar nome e afiliação dos membros do comitê editorial; } \\
\text { divulgar anualmente a nominata dos revisores; publicar mínimo } \\
\text { de dois números por ano; informar dados completos dos } \\
\text { artigos; e constar endereço de pelo menos um dos autores. }\end{array}$ & $\begin{array}{l}\text { ISSN; ter no mínimo } 2 \text { edições/ano; índice } \\
\text { de atraso no máximo igual a } 0,5 ; 3 \text { ou mais } \\
\text { anos de existência; e ter no mínimo um dos } \\
\text { indexadores definidos no documento da área. }\end{array}$ \\
\hline B4 & $\begin{array}{l}\text { Atender aos critérios do estrato } \mathrm{B} 5 \text { e: } \\
\text { Ter revisão por pares; edições atualizadas; } \\
\text { e normas de submissão. }\end{array}$ & $\begin{array}{l}\text { ISSN; ter no mínimo } 2 \text { edições/ano; índice de atraso } \\
\text { no máximo igual a 0,5; } 2 \text { ou mais anos de existência. }\end{array}$ \\
\hline B5 & Ter ISSN e ter periodicidade definida. & $\begin{array}{l}\text { ISSN; ter no mínimo } 2 \text { edições/ano e } \\
\text { no máximo um ano de atraso. }\end{array}$ \\
\hline $\mathrm{C}$ & Periódicos não atendem aos critérios para ser B5. & Não são classificados. \\
\hline
\end{tabular}

Fonte: CAPES (2013a; 2015b)

\subsubsection{Journal Citation Report (JCR)}

O JCR é a ferramenta de avaliação e indicador bibliométrico mais amplamente utilizado na literatura científica (FINARDI, 2013). O JCR disponibiliza o indicador 'Fator de Impacto' (FI). Este foi mencionado pela primeira vez em 1955, em um artigo publicado na Science sobre índices de citações para avaliar a importância, ou impacto intelectual, de um trabalho científico (GARFIELD, 2006). No entanto, foi apenas em 1963 que Irving Sher e Eugene Garfield criaram o Journal Impact Factor (JIF). O FI foi ganhando importância e passou a ser usado para aferir a qualidade dos periódicos, o impacto dos periódicos e dos autores. Assim, o FI foi utilizado pela Science Citation Index (SCI) como primeira ferramenta de ranqueamento de publicações, antes mesmo da criação do JCR (GARFIELD, 2006). O JCR divulga o FI de um periódico, com base no valor adotado pela Science Citation Index (COURA; WILLCOX, 2003; FERREIRA, 2015).

O que é o fator de impacto de um periódico? É uma medida de importância aferida pelas citações aos artigos que publicou em anos anteriores. Por exemplo, para calcular o FI de um periódico em 2014, divide-se o número de citações dos artigos publicados no periódico nos dois anos anteriores, pelo número de citações a esses artigos em 2014 - ver Garfield (2006). É comum utilizar-se o FI a dois anos em decorrência do estudo de Garfield (1972) que concluiu que os 
artigos são mais citados nos dois anos seguintes ao da publicação, correspondendo de $21 \%$ a $25 \%$ do total das citações que receberão.

Anualmente, o JCR publica três indicadores: o índice de citação imediata, a meia-vida das citações (MV) e o FI, mencionado anteriormente (STREHL, 2005). O índice de citação imediata está relacionado com a rapidez com que um artigo é citado durante o ano de publicação, sendo que quanto menor o tempo, maior será o valor. Já a MV das citações está relacionada ao ritmo de envelhecimento da literatura, ou seja, constatar quando um trabalho atinge metade de sua vida útil. Para isso, é levado em consideração o tempo, em anos, que um periódico leva para receber $50 \%$ das citações.

\subsubsection{A Scopus}

A Scopus foi lançada pela Elsevier em 2004 (ARCHAMBAULT et al., 2009) e tem vindo a afirmar-se como uma referência a fornecer indicadores de impacto e citações. Seu índice H começa a ser mais utilizado pelas universidades na avaliação dos periódicos. A Scopus é uma base de dados de ampla cobertura (LEYDESDORFF et al., 2015) que engloba revistas científicas, livros e anais de eventos (RODRIGUES; OLIVEIRA, 2012).

Para inclusão na base da Scopus o periódico tem de cumprir alguns critérios como: a) os artigos terem revisão pelos pares em sistema de blind review (revisão cega - designação que revela que nem os autores conhecem a identidade dos revisores, nem os revisores sabem quem são os autores) e prover uma descrição disponível ao público deste processo; b) o periódico deve ser publicado numa base regular e ter ISSN; c) proporcionar conteúdo relevante que pode ser lido internacionalmente, ou seja, usar caracteres romanos e ter resumos e títulos em língua inglesa; d) disponibilizar a ética de publicação e declaração de publicação negligenciada (ELSEVIER, 2015a).

Em 2015, o Scopus Content Selection and Advisory Board (CSAB) - um conselho internacional de cientistas, pesquisadores e bibliotecários que representam as principais disciplinas científicas - introduziu mudanças no Scopus. Para inclusão no Scopus os periódicos são reavaliados usando seis métricas de qualidade, (ELSEVIER, 2015a). A base Scopus também alargou passando a incluir artigos publicados entre 1970 e 1996 - em resposta a crítica de Goodman e Deis (2004) - e a expansão da Scopus Livros (ELSEVIER, 2015b).

\subsubsection{A SCIELO}

A biblioteca SciELO (Scientific Electronic Library Online) surgiu em 1998, para ajudar a divulgar e dar visibilidade à ciência produzida no Brasil, onde, na sua grande maioria, os periódicos brasileiros não estão indexados nas bases internacionais como a WoS ou a Scopus. A SciELO inclui uma coleção multidisciplinar de revistas científicas nacionais disponibilizadas em acesso aberto (open access). Desde o início, para além da disponibilização dos artigos, a SciELO objetivou contribuir para a melhoria da qualidade dos periódicos (SANTOS ; NORONHA, 2013). Outro objetivo da SciELO foi o de criar uma base de dados bibliométricos com o intuito de produzir indicadores de desempenho similares aos gerados pela Thomson Scientific's Web of Science e JCR (MENEGHINI; PACKER, 2007).

A SciELO se propôs solucionar de modo simultâneo a publicação dos textos completos na internet, a sua indexação e a medição do seu uso e impacto (PACKER, 2002). Ao longo do seu desenvolvimento, a Rede SciELO foi incorporando publicações de outros países, tornando-se um padrão de qualidade para os periódicos que indexava (PACKER et al., 2014). Atualmente, o Qualis já incorpora a possibilidade de usar os indicadores de impacto gerados pela SciELO.

\section{MÉtodo}

Para avaliar o impacto da alteração do Qualis para o quadriênio 2013-2016 na contabilização da pontuação das publicações dos pesquisadores, efetuou-se um estudo de natureza descritiva sustentado em dados coletados de fontes secundárias. Este estudo requereu a definição de uma amostra aleatória de pesquisadores, a contabilização da sua publicação científica coletada dos currículos Lattes dos pesquisadores e a análise das pontuações segundo os Qualis 2010-12 e Qualis 2013-16. 


\subsection{Amostra}

A delimitação da amostra para o estudo envolveu alguns procedimentos que especificou. Primeiro, no portal de internet da CAPES foi acessado o Sistema de Disseminação de Informações que disponibiliza em formato Excel os resultados da avaliação trienal de 2013 (CAPES, 2013b). Com a aplicação de um filtro, delimitou-se a pesquisa a apenas PPGs em Administração, tais como: Administração, Administração das micro e pequenas empresas, Administração de empresas, Administração de organizações, Administração e controladoria, Administração e desenvolvimento empresarial, Administração e negócios, Administração pública, e Administração universitária. Com esse procedimento, foram identificados os PPGAs e os seus conceitos CAPES.

Em seguida foi acessado o Caderno de Indicadores 2013 da CAPES (2015c). Selecionando o ano de 2012 - o último ano disponível - realizou-se a pesquisa por instituição e pela área de 'Administração, Ciências Contábeis e Turismo'. Para cada instituição, no arquivo denominado 'CD' referente ao corpo docente, extraiu-se o nome dos docentes permanentes (DP) de cada organização no ano de 2012.
O terceiro procedimento envolveu o cálculo do tamanho da amostra, com o número de DP por instituição e o número total de instituições. A fórmula para determinação da amostra é representada da seguinte forma: $n=$ amostra calculada; $N=$ população; $Z=$ variável normal padronizada associada ao nível de confiança; $p=$ verdadeira probabilidade do evento e $e=$ erro amostral. Os parâmetros fixados foram: margem de erro projetado para o intervalo do resultado de 5\%, nível de confiança do intervalo de $95 \%$, proporção máxima admitida para a variável principal da pesquisa de $50 \%$, e fator não aplicado de correção para a população finita, dado que o tamanho da população equivale ao padrão de população infinita.

$$
n=\frac{N \cdot Z^{2} \cdot p \cdot(1-p)}{Z^{2} \cdot p \cdot(1-p)+e^{2} \cdot(N-1)}
$$

Estima-se a necessidade de uma amostra de aproximadamente 97 indivíduos, para uma análise significativa e relevante. Fora, identificados 21 cursos com conceito 3, 40 com conceito 4, 14 com conceito 5, e apenas 2 com o conceito 6 (FGV-RJ e UFMG) e 7 (na FEA-USP e FGV-SP). O tamanho e distribuição da amostra foram definidos face ao número de docentes permanentes (DP) nos programas em 2012 (Tabela 3).

Tabela 3: Distribuição da amostra por conceito CAPES do PPGA

\begin{tabular}{|c|c|c|c|c|}
\hline Número de cursos & Conceito CAPES & Número de DPs & $\begin{array}{c}\text { Número de DPs } \\
\text { analisados }\end{array}$ & $\begin{array}{c}\text { Representação } \\
\text { na amostra }\end{array}$ \\
\hline 21 & 3 & 253 & 22 & $22,7 \%$ \\
\hline 40 & 4 & 547 & 46 & $47,4 \%$ \\
\hline 14 & 5 & 244 & 19 & $19,6 \%$ \\
\hline 2 & 6 & 46 & 4 & $4,1 \%$ \\
\hline 79 & 7 & 81 & 6 & $6,2 \%$ \\
\hline
\end{tabular}

Nota: DP - Docentes Permanentes.

Fonte: Dados da pesquisa

\subsection{Coleta de Dados}

Após a determinação do tamanho da amostra e sua distribuição, elaborou-se uma lista de todos os professores e foi realizado um sorteio aleatório. Para isso utilizou-se o software disponibilizado no site: www. sorteador.com.br. Para cada instituição foram sorteados cinco números, conforme ordem da listagem dos DP por instituição. Como este estudo exige analisar as pontuações dos artigos publicados, se um professor sorteado não tivesse artigos publicados em 2014, seguia-se para o próximo sorteado.e procedimento permitiu obter uma amostra realmente aleatória, garantindo a representatividade de pesquisadores por conceito de PPGA.

Determinados quais os currículos que integram a amostra, iniciou-se a coleta de dados. Na plataforma Lattes, foram acessados os currículos de cada um dos pesquisadores na amostra e extraídos apenas os dados dos artigos publicados em 2014. Esse contexto é relevante na medida em que em 2014 os pesquisadores apenas conheciam o Qualis então vigente para o triênio 20102012 e ao ser alterado para o Qualis 2013-2016 teve 
efeitos retroativos a 2013 e 2014. Ou seja, nesta etapa coletou-se o número de artigos publicados, e indicação específica do periódico em que cada artigo foi publicado.

O último procedimento envolveu a classificação dos artigos que cada pesquisador publicou conforme os Qualis 2012 e Qualis 2014, seguindo a informação disponibilizada na plataforma Sucupira. Periódicos não classificados na área de 'Administração, contábeis e turismo' para qualquer um dos períodos, foram excluídos. Com esses procedimentos obteve-se 325 artigos que foram publicados em 2014 pelos 97 pesquisadores. Para análise, foram usadas estatísticas descritivas baseadas em contagens, porcentagens e médias. Não se fez uso de estatísticas multivariadas já que não se pretendia fazer inferências adicionais.

\section{Resultados}

A primeira análise incidiu na observação da distribuição dos 325 artigos segundo os estratos Qualis (Tabela 4) dos periódicos. Segundo o Qualis 2010-12, 71\% dos artigos foram publicados em periódicos entre os estratos B1, B2 e B3. No entanto, com o novo Qualis 2013-16, $7 \%$ destes ficaram entre os estratos B2, B3 e B4. Ou seja, houve uma variação substancial por aplicação retroativa do novo Qualis. Em suma, 67\% dos artigos foram reclassificados com a aplicação do novo Qualis; sendo que $56 \%$ foram reclassificados para um estrato inferior e apenas $11 \%$ para estratos superiores. É interessante salientar que desses $11 \%$ que subiram de estrato, $58 \%$ se referem a artigos que haviam sido publicados em periódicos com estratos mais baixos, B4 e B5 e que foram reclassificados subindo no Qualis para o quadriênio 2013-2016.

Tabela 4: Análise comparativa segundo a classificação Qualis dos periódicos

\begin{tabular}{|c|c|c|c|c|c|c|c|c|c|}
\hline & A1 & A2 & B1 & B2 & B3 & B4 & B5 & $\mathbf{C}$ & TOTAL \\
\hline Quant. artigos em cada estrato (Qualis 2010-12) & 13 & 51 & 77 & 74 & 77 & 15 & 15 & 3 & 325 \\
\hline$\%$ & 4 & 16 & 24 & 23 & 24 & 5 & 5 & 1 & $100 \%$ \\
\hline $\begin{array}{l}\text { Quant. artigos em cada estrato (por } \\
\text { reclassificação no Qualis 2013-16) }\end{array}$ & 9 & 45 & 40 & 56 & 101 & 55 & 13 & 6 & 325 \\
\hline$\%$ & 3 & 14 & 12 & 17 & 31 & 17 & 4 & 2 & $100 \%$ \\
\hline
\end{tabular}

Fonte: Dados da pesquisa

$\mathrm{Na}$ análise dos números absolutos de artigos (Figura 1) segundo o estrato Qualis fica evidente que as quedas nos estratos A1, A2, B1 e B2 foram absorvidas nos estratos B3 e B4.



Qualis 2010-2012

Qualis 2013-2016

Figura 1: Comparativo das publicações conforme Qualis 2010-12 e 2013-16

Fonte: Dados da pesquisa

A segunda análise incidiu sobre a mobilidade. Ou seja, observando as posições relativas das publicações no estrato Qualis 2010-12 e o reposicionamento por aplicação do Qualis 2013-16. A Tabela 5 apresenta os resultados. Na leitura da tabela, no eixo horizontal, a primeira linha apresenta a origem da classificação dos periódicos onde os artigos foram publicados, em números absolutos. No eixo vertical, na primeira coluna, números totais relacionados à sua reclassificação. Demais células revelam o posicionamento da classificação dos periódicos onde estes artigos foram publicados, sob a nova regra. Na diagonal, o número de artigos publicados em revistas que não sofreram alterações de classificação. Acima da diagonal, os artigos publicados em periódicos que ascenderam no novo Qualis, e abaixo da diagonal artigos publicados em periódicos que desceram a pontuação com o novo Qualis. Por exemplo, dos 77 artigos B1 no Qualis 2010-12, somente 22 mantiveram a classificação com o Qualis 2013-16, 9 subiram para A2, mas 43 desceram para B2 e 3 para B3. Portanto, sob as novas regras, o estrato $\mathrm{B} 1$ passou a ter 40 artigos - resultado dos 22 que se mantiveram mais os 16 que desceram do nível A2, um que subiu do B2 e um que subiu do B4. 
Tabela 5: Reclassificação dos artigos por aplicação do Qualis 2013-2016

\begin{tabular}{|c|c|c|c|c|c|c|c|c|c|c|}
\hline & & & \multicolumn{8}{|c|}{ Número de ARTIGOS E ClassificaÇão QuALIS 2010-12 } \\
\hline & & & 13 & 51 & 77 & 74 & 77 & 15 & 15 & 3 \\
\hline & & & $\mathrm{A} 1$ & A2 & B1 & B2 & B3 & B4 & B5 & C \\
\hline \multirow{8}{*}{ 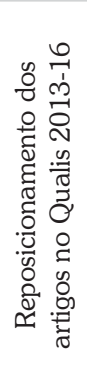 } & 9 & A1 & 9 & & & & & & & \\
\hline & 45 & A2 & 4 & 32 & 9 & & & & & \\
\hline & 40 & B1 & & 16 & 22 & 1 & & 1 & & \\
\hline & 56 & B2 & & 3 & 43 & 8 & 2 & & & \\
\hline & 101 & B3 & & & 3 & 56 & 31 & 9 & 1 & 1 \\
\hline & 55 & B4 & & & & 5 & 36 & 4 & 9 & 1 \\
\hline & 13 & B5 & & & & 4 & 7 & 1 & 1 & \\
\hline & 6 & C & & & & & 1 & & 4 & 1 \\
\hline
\end{tabular}

Fonte: Dados da pesquisa

Esta análise foi complementada com a indicação de percentuais de mudanças (Tabela 6). Observando por estrato, verificou-se que para o estrato $\mathrm{B} 1$ houve uma queda de $60 \%$, para B2 queda de $88 \%$ e B3 de
$57 \%$. Alguns periódicos caíram três posições no novo Qualis. Nos estratos A1 e A2, 69\% e 63\% dos artigos, respectivamente, mantiveram a pontuação.

Tabela 6: Mobilidade da qualificação com aplicação do novo Qualis

\begin{tabular}{|c|c|c|c|c|c|c|c|c|c|}
\hline & A1 & A2 & B1 & B2 & B3 & B4 & B5 & C \\
\hline \% de artigos que perderam posições & $31 \%$ & $37 \%$ & $60 \%$ & $88 \%$ & $57 \%$ & $7 \%$ & $27 \%$ & 0 \\
\hline \% de artigos que se mantiveram & $69 \%$ & $63 \%$ & $28 \%$ & $11 \%$ & $40 \%$ & $27 \%$ & $7 \%$ & $33 \%$ \\
\hline \% de artigos que ganharam posições & 0 & 0 & $12 \%$ & $1 \%$ & $3 \%$ & $67 \%$ & $66 \%$ & $67 \%$ \\
\hline & $100 \%$ & $100 \%$ & $100 \%$ & $100 \%$ & $100 \%$ & $100 \%$ & $100 \%$ & $100 \%$ \\
\hline
\end{tabular}

Fonte: Dados da pesquisa

Por fim, a terceira análise envolveu entender o impacto das mudanças do Qualis nas pontuações dos professores nos PPGAs e, assim, previsivelmente nos próprios programas. Como pode ser notado, o conceito CAPES dos PPGAs se dá em função de diversos indicadores, pelo que analisar as pontuações das publicações é uma métrica parcial e não permite prever eventuais mudanças nos conceitos dos programas que possam vir a ocorrer.

A análise dos dados da amostra (Figura 2) revela que ao todo, $23,7 \%$ dos docentes permanentes (23 DPs) não teve alteração na pontuação, $7,2 \%$ teve um aumento na pontuação (7 DPs) e uma maioria de $69,1 \%$ teve uma redução na pontuação por aplicação do novo Qualis (67 DPs). Assim, para entender melhor o impacto, foi feita a análise por conceito CAPES do programa e observou-se que as perdas se refletem em todos os programas, ainda que em programas de conceito 4 e 5 a perda seja parcialmente compensada com algum ganho. Também, a menor média de perda, $-32,5$ pontos, se deu nos programas conceito 7 e a maior média de perda, $-49,2$ pontos, em programas com conceito 5. Em termos de percentegem, o conceito que mais teve docentes com perdas foi o $3 \mathrm{com} 77,3 \%$. Em números absolutos, destaca-se o conceito $4 \mathrm{em}$ que 31 docentes tiveram perdas. Os DPs do conceito 6 foram os menos impactados pelas mudanças no Qualis, atingindo em torno de $50 \%$ deles. Uma análise mais individualizada por PPGA não é viável por ser usada apenas uma amostra de todos os DPs.

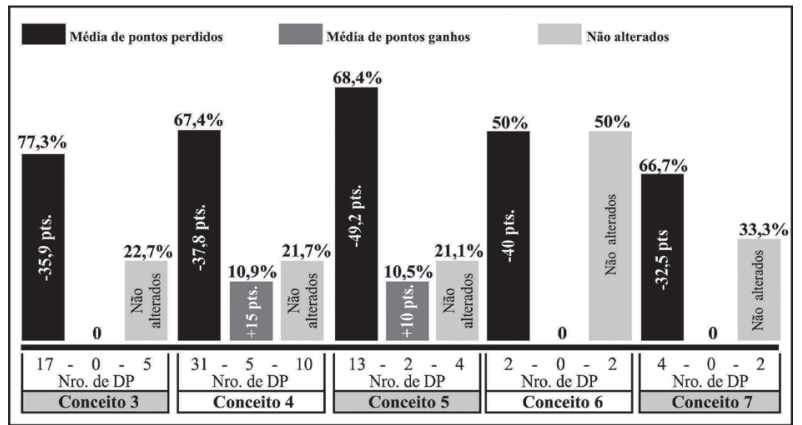

Nota: DP: docentes permantentes

Figura 2: Impacto da alteração no Qualis na pontuação dos docentes permanentes

Fonte: Dados da pesquisa 


\section{Discussão}

A avaliação do desempenho científico dos professores pesquisadores utilizando sistemas baseados em métricas quantitativas de impacto dos periódicos é prática comum (STAHL et al., 1988; OLIVEIRA et al., 2015; TRUEGER et al., 2015), seja usando o fator de impacto, a indexação no SSCI (Social Science Citation Index) ou na Scopus (FERREIRA, 2015). Também no Brasil, sob a coordenação da CAPES, está instituído um sistema similar que tem no Qualis o ranqueamento das publicações e que norteia não apenas as avaliações dos pesquisadores (importante, por exemplo, para atribuição de bolsas de fomento ou de produtividade), mas também, dos programas em especial dos stricto sensu (NASCIMENTO; BEUREN, 2011; FERREIRA, 2015; OLIVEIRA et al., 2015).

Neste artigo serão debatidos e apresentados os indexadores mais comumente utilizados como o FI e a Scopus. E, face à recente alteração do Qualis, que foi apresentada em 2015 para o quadriênio 2013 a 2016, porém com efeitos retroativos a 2013 e 2014, analisou-se qual o impacto da mudança do Qualis na produção intelectual de uma amostra aleatória de pesquisadores em Administração. Os dados, analisados com técnicas descritivas, suportam o objetivo de mostrar as alterações nas pontuações dos pesquisadores. Previsivelmente, este impacto também se fará refletir nos conceitos CAPES dos programas stricto sensu, mas está fora do escopo estimar as possíveis alterações no conceito CAPES dos PPGAs em função exclusiva das alterações no Qualis.

Entender a forma como pesquisadores e programas são avaliados pelas agências reguladoras é crucial. Os dirigentes desenvolvem ações que visam à melhoria dos programas atendendo a forma como essas ações serão avaliadas. Os pesquisadores desenvolverão esforços diferenciados de acordo com os critérios segundo os quais serão avaliados. Os estudantes podem usar as avaliações na seleção para qual PPGA se candidatar. As agências reguladoras definem o apoio que prestam aos programas servindo-se dos resultados das avaliações. A sociedade confia que as avaliações são indicadores fidedignos da qualidade relativa dos programas e educação fornecida. Os mecanismos de avaliação funcionam, assim, como sistemas de incentivos que direcionam as ações dos agentes envolvidos.
Ou seja, a avaliação e a forma como é feita, importa a todos os agentes.

Há, também, consequências para os próprios periódicos. A avaliação dos periódicos é um sinalizador da sua qualidade - atraindo, ou afastando, as submissões dos pesquisadores. Por exemplo, o Qualis 2013-2016 rebaixou alguns periódicos nacionais, valorizando mais a utilização de métricas como a indexação em bases internacionais e os fatores de impacto. O risco para os periódicos rebaixados é diminuir o número de submissões, vendo assim, comprometidos os seus planos de melhoria. Em alguns casos podem perder o suporte das suas organizações, perdendo a academia um veículo importante de disseminação do conhecimento. No caso do Brasil, a perda pode ser mais significativa porque se perderiam periódicos de divulgação do conhecimento em língua portuguesa, em relativa valorização das publicações internacionais - mais afastadas do público nacional e escritas em inglês. Importa, assim, analisar cuidadamente quaisquer alterações no quadro institucional regulador (não exclusivamente do Qualis) pelos efeitos que induzem nos pesquisadores, programas e periódicos. Há, assim, implicações para a prática e este estudo contribui para o debate sobre os mecanismos de avaliação da produção intelectual ao analisar quantitativamente as alterações nas pontuações que foram introduzidas por uma mudança no sistema Qualis para o quadriênio 2013-2016. Neste caso específico, a particularidade é que o novo Qualis foi implementado com efeitos retroativos, assim frustrando as expectativas dos pesquisadores sobre pontuação quando selecionaram os periódicos onde submeteram os seus artigos entre 2013 e 2014 (ver a crítica de Rocha-e-Silva (2009) a falta de debate da CAPES).

Os resultados da análise empírica são taxativos a apontar uma redução geral nas pontuações (ou seja, na avaliação dos pesquisadores) em resultado das alterações no Qualis. Numa amostra representativa de docentes permanentes dos programas stricto sensu em Administração, concluiu-se que, no Qualis anterior (2010-2012), as publicações dos pesquisadores se concentravam em periódicos entre os estratos B1, B2 e B3. Em comparação ao novo Qualis, passaram a se concentrar, predominantemente, no estrato B3. Esta é uma evidência forte da desqualificação da produção científica dos docentes. 
Ainda assim, há casos em que os pesquisadores foram beneficiados com as alterações e, em especial, os que publicaram menos artigos e, fundamentalmente, em periódicos que eram anteriormente classificados em estratos B4, B5 e C (análise não incluída neste artigo, mas disponível dos autores). Se um dos objetivos da mudança das regras do Qualis era contribuir para a melhoria da qualidade das publicações, então fica pouco claro como esta mudança realmente é realizada.

Os resultados também permitem breves inferências sobre a avaliação dos PPGA. Especificamente, foi feita uma análise de como os programas de pós-graduação stricto sensu seriam afetados apenas olhando a pontuação dos seus docentes permanentes. Os resultados mostram que há uma perda significativa na pontuação dos PPGAs por aplicação dos novos critérios do Qualis. As consequências parecem impactar mais substancialmente os programas com conceitos 4 e 5. A perda apenas é comparativamente menor nos programas conceito 7 , ainda que também estes tenham perdas substanciais. É necessária pesquisa futura a este respeito, para entender como os programas podem ultrapasssar estas perdas com outras ações de modo a não terem os seus conceitos rebaixados.

As alterações introduzidas ao Qualis para o quadriênio 2013-16 geraram uma discussão exaltada em inúmeros eventos nacionais e teve sessão dedicada no Enanpad 2015. Em virtude da pouca receptividade ao novo Qualis foram feitos alguns ajustes a posteriori. Em parte, o problema pode ter residido no desconhecimento das regras e critérios que iriam emergir sobre a classificação dos periódicos para 2013 e 2014, e o novo Qualis, quando apresentado em 2015, teve efeitos retroativos. Ou seja, os pesquisadores que publicaram artigos em 2014 selecionaram os periódicos onde submeteram com base numa lista Qualis que era vigente à época, mas que veio a ser posteriormente alterada. Viram, assim, as suas expectativas de pontuação frustradas.

As potenciais distorções induzidas por sistemas de avaliação da produção intelectual que não sejam concebidos são conhecidas. São distorções que poderão, por exemplo, privilegiar certos grupos (por exemplo, valorizando os periódicos de certas áreas de conhecimento), ou algumas universidades e programas sobre outros, criando percepções errôneas sobre a qualidade dos programas. Outra distorção é a que inviabiliza a mobilidade dos programas. Por exemplo, dificultando desproporcionalmente a progressão de alguns programas nos conceitos CAPES. Esta não é uma questão menor porque aos diferentes conceitos CAPES corresponde um nível diferenciado de financiamento (número de bolsas) dos estudantes de mestrado e de doutorado (OLIVEIRA et al., 2015). Assim, não surpreende que o Qualis, como sistema de ranqueamento, e pelas suas implicações, seja alvo de críticas (ver, por exemplo, ROCHA-E-SILVA, 2009; TREIN; RODRIGUES, 2011).

\subsection{Limitações e Pesquisa Futura}

Este estudo tem limitações. Primeiro, o escopo do nosso estudo não incluiu todos os aspectos que têm vindo a alterar-se no quadro institucional regulador e que impactam as avaliações de pesquisadores e de programas stricto sensu. Assim, enquanto se conseguiu captar uma ideia geral do impacto sobre os programas (os PPGAs) na dimensão de produção intelectual, o nosso estudo apenas incidiu sobre uma amostra dos docentes permanentes. Um estudo mais extenso seria adequado para melhor entendimento de, por exemplo, qual o impacto real sobre cada programa das recentes alterações do Qualis. Importa notar que a produção científica dos pesquisadores inclui outros aspectos para além dos artigos, embora estes talvez sejam o componente mais saliente (GREENWOOD, 1998; HARZING, 2013; FERREIRA, 2015). A contribuição científica dos pesquisadores inclui, também, as orientações, trabalhos técnicos, livros, apresentações em conferências, patentes, trabalhos técnicos, entre outros.

Pesquisa futura merece ser realizada para promover o debate sobre as formas de avaliação dos programas e dos pesquisadores. Os critérios definidos não são inócuos e afetam, por exemplo, a capacidade de atrair recursos financeiros, mas, também, sinalizam qualidade dos programas e assim contribuem para atrair melhores estudantes e professores mais prolíferos. Talvez este debate necessite ser realizado de forma alargada a todos os agentes da academia $e$ abranger aspectos como: Qual o caminho futuro da avaliação da produção intelectual? Quais os sistemas de incentivos que auxiliarão pesquisadores a contribuir para o progresso do sistema nacional de educação $e$ ciência? Qual o impacto sobre a produção nacional 
da tendência para seguir métricas usadas em outros países? Como manter os periódicos nacionais contribui para manter a língua portuguesa?

Pesquisa futura pode, também, contribuir para elucidar o impacto das alterações no Qualis sobre os periódicos. O Qualis 2013-16 permite antever uma tendência futura de relativa desvalorização das publicações em periódicos nacionais. Mas, qual o efeito sobre o número de periódicos brasileiros? Se uma das intenções que presidiu ao novo Qualis foi diminuir a homogeneidade que os periódicos estavam alcançando - e que dificultava o atendimento da regra da distribuição deles - importa fazer o acompanhamento dos esforços dos periódicos para que melhorem. A dificuldade passará por atrair bons artigos dos pesquisadores que buscarão publicar nos periódicos em estratos Qualis mais altos. Ou seja, há um risco eventual de baixar a qualidade efetiva das publicações em periódicos nacionais, tolhendo as ambições de ter periódicos nacionais internacionalmente reputados.

\section{Considerações Finais}

Os sistemas de avaliação que as organizações e instituições utilizam são direcionadores importantes da evolução do sistema científico do país. Indivíduos e organizações agem e reagem em função dos quadros de incentivos. No Brasil, tanto pesquisadores como universidades são sensíveis às métricas definidas pela CAPES nas suas avaliações. E, sendo a produção científica uma das vertentes internacionalmente mais valorizadas do desempenho docente (MILLER et al., 2011; HARZING, 2013), todos os sistemas, métodos e métricas que balizem a forma como a avaliação é realizada têm impacto substancial na produção científica.

As modificações do Qualis ocasionaram alterações na classificação da maioria dos periódicos identificados neste estudo. Nota-se, por exemplo, o impacto gerado pela reclassificação de muitos periódicos para estratos Qualis inferiores aqueles em que os periódicos estavam posicionados no Qualis anterior. Na realidade, uma parte substancial dos periódicos nacionais foi reposicionado em estrato Qualis inferior. A parcela que ascendeu na classificação refere-se aos classificados em estratos inferiores, portanto, este acréscimo se deve em parte pela absorção dos periódicos que tiveram queda na sua classificação. Esta queda na classificação dos periódicos teve impacto bastante generalizado na pontuação dos pesquisadores. Agrupando os pesquisadores pela classificação dos PPGAS, programas em todos os conceitos tiveram pesquisadores com pontuação Qualis inferior ao que se estimava, tendo por base os critérios Qualis anteriores. Esta evidência é relevante na medida em que tem implicações na avaliação dos pesquisadores e dos próprios PPGs.

A análise ao sistema nacional mostra que as diversas atualizações no Qualis e as mudanças que têm marcado o sistema nacional científico têm contribuído para o aperfeiçoamento e rigor científico. A maior regulamentação tem contribuído para melhorias nos periódicos que se podem repercutir em todo o sistema de ensino superior. Talvez a tendência futura passe por maior internacionalização, inclusive com a busca por publicações internacionais de impacto. O Brasil já dispõe de um dos sistemas institucionais mais sofisticados (MACCARI et al., 2008) e, independentemente dos critérios estabelecidos para o Qualis, é previsível que a melhoria da produção científica continue a ocorrer, tal como em outras economias emergentes, por exemplo, Asiáticas.

\section{REFERÊNCIAS}

ADAM, D. Citation analysis: The counting house. Nature, [S.l.], v. 415, p. 726-729, 2002.

ALCADIPANI, R. Academia e fábrica de sardinhas. Organizações e Sociedade, [S.I.], v. 18, n. 57, p. 345348, 2011.

ARCHAMBAULT et al. Comparing bibliometric statistics obtained from the Web of Science and Scopus. Journal of the American Society for Information Science and Technology, v. 60, n. 7, p. 1.320-1.326, 2009.

BAKKALBASI et al. Three options for citation tracking: Google Scholar, Scopus and Web of Science. Biomedical Digital Libraries, [S.I.], v. 3, n. 1, p. 7-15, 2006.

BAR-LLAN, J. Which h-index? A comparison of WoS, Scopus and Google Scholar. Scientometrics, [S.l.], v. 74, n. 2, p. 257-271, 2008. 
CAPES. Documento de área e Comissão da Trienal 2013. Brasília, DF, Brasil, 2013a.

CAPES. Relatório de avaliação trienal 2013.

Fundação Capes, Ministério da Educação. Brasília, DF, Brasil: Fundação Capes, Ministério da Educação, 2013 b.

CAPES. História e missão. [2015a]. Disponível em: <http://www.capes.gov.br/historia-e-missao > . Acesso em: 5 nov. 2015.

\section{CAPES. Relatório do processo de classificação de} periódicos: Área de Administração, Ciências Contábeis e Turismo Quadriênio 2013-2016. Brasília, DF, Brasil: Fundação Capes, Ministério da Educação, 2015b.

CAPES. Caderno de Indicadores. [2015c]. Disponível em: <http://conteudoweb.capes.gov.br/conteudoweb/Cad ernoAvaliacaoServlet?acao $=$ filtraArquivo $\&$ ano $=2012 \& \mathrm{co}$ digo_ies $=\& a r e a=27>$. Acesso em: 6 nov. 2015.

COELHO et al. The use and misuse of the "impact factor" as a parameter for evaluation of scientific publication quality: a proposal to rationalize its application. Brazilian Journal of Medical and Biological Research, [S.l.], v. 36, n. 12 , p. 1.605-1.612, 2003.

COURA, J.; WILLCOX, L. Impact factor, scientific production and quality of Brazilian medical journals.

Memórias do Instituto Oswaldo Cruz, [S.l.], v. 98, p. 293-298, 2003.

ELSEVIER. Content policy and selection. [2015a]. Disponível em: < https:/www.elsevier.com/solutions/ scopus/content/content-policy-and-selection > . Acesso em: 24 dez. 2015.

\section{ELSEVIER. Scopus 2015 Review, Part 1: More} content, but not at the expense of quality. [2015b]. Disponível em: <http://blog.scopus.com/posts/Scopus2015-review-part-1-more-content-but-not-at-the-expenseof-quality >. Acesso em: 24 dez. 2015.

FALASTER, C.; FERREIRA, M. P.; SERRA, F. R. The research productivity of new Brazilian PhDs in management: a few "star" performers outshine a mass of low performers. Management Research: The Journal of the Iberoamerican Academy of Management, [S.I.], v. 14, n. 1, p. 60-84, 2016.
FERREIRA, M. Periódicos e rankings de periódicos em Administração. Revista Pensamento Contemporâneo em Administração, [S.l.], v. 9, n. 2, p. 1-16, 2015.

FERREIRA, M. P.; FALASTER, C. Uma Análise Comparativa dos Fatores de Rejeição nos Periódicos de Diferentes Estratos de Administração. Revista de Administração Contemporânea, [S.1.], v. 20, n. 4, p. 412-433, 2016.

FIATES, G.; SERRA, F; MARTINS, C. A aptidão dos pesquisadores brasileiros pertencentes aos programas de pós-graduação stricto sensu em Administração para pesquisas quantitativas. Revista de Administração, [S.l.], v. 49, n. 2, p. 291-306, 2014.

FINARDI, U. Correlation between journal impact factor and citation performance: An experimental study. Journal of Informetrics, [S.I.], v. 7, n. 2, p. 357-370, 2013.

GARFIELD, E. Citation analysis as a tool in journal evaluation. Science, [S.l.], v. 178, p. 471-479, 1972.

GARFIELD, E. The history and meaning of the journal impact factor. Jama, [S.l.], v. 295, n. 1, p. 90-93, 2006.

GASPAR, M.; CAMPOS, D. Estudo da produtividade de doutores egressos do PPGA. Revista da Faculdade de Administração e Economia, [S.l.], v. 6, n. 2, p. 14-32, 2015.

GAZZOLA, A. Desigualdade é a marca da educação superior na América Latina e Caribe. Jornal da Ciência da Sociedade Brasileira Para o Progresso da Ciência (SBPC), v. XXII, n. 624, 2008.

GOODMAN, D.; DEIS, L. Web of Science (2004 version) and Scopus. The Charleston Advisor, [S.l.], v. 6, n. 3, p. 5-21, 2004.

GREENWOOD, C. Publish or perish: The ethics of publishing in peer-reviewed journals, Media Information Australia, [S.I.], v. 68, p. 29-35, 1998.

HARZING, A. W. The publish or perish book: Your guide to effective and responsible citation analysis. Melbourne, Australia: Tarma Software Research Pty Ltd., 2013. 
HECHT, F; HECHT, B.; SANDBERG, A. The journal "impact factor": A misnamed, misleading, misused measure. Cancer Genet Cytogenet, [S.l.], v. 104, n. 2, p. 77-81, 1998.

HIRSCH, J. An index to quantify an individual's scientific research output. Proceedings of the Natlonal Academy Science of the United States of America, [S.I.], v. 102, n. 46, p. 16.569-16.572, 2005.

KUENZER, A.; MORAES, M. Temas e tramas na pósgraduação em educação. Educação \& Sociedade, [S.l.], v. 26, n. 93, p-1.341-1.362, 2005.

LEYDESDORFF, L.; MOYA-ANEGÓN, F; GUERRERO-

BOTE, $V$. Journal maps, interactive overlays, and the measurement of interdisciplinarity on the basis of Scopus data (1996-2012). Journal of the Association for Information Science and Technology, [S.I.], v. 66, n. 5, p. 1.001-1.016, 2015.

MACCARI et al. Sistema de avaliação da pós-graduação da CAPES: pesquisa-ação em um programa de pósgraduação em Administração. Revista Brasileira de Pós-Graduação, v.5, n.9, p.171-205, 2008.

MACCARI et al. A gestão dos programas de pósgraduação em administração com base no sistema de avaliação da CAPES. REGE Revista de Gestão, [S.I.], v. 16, n. 4, p. 1-16, 2009.

MACCARI et al. Proposta de modelo de gestão de programas de pós-graduação na área de Administração a partir dos sistemas de avaliação do Brasil (Capes) e dos Estados Unidos (AACBS). Revista de Administração da USP, [S.I.], v. 49, n. 2, p. 369-383, 2014.

MENEGHINI, R.; PACKER, A. Is there science beyond English? Initiatives to increase the quality and visibility of non-English publications might help to break down language barriers in scientific communication. EMBO Reports, [S.l.], v. 8, n. 2, p. 112-116, 2007.

MIGUEL, S. Revistas y producción científica de América Latina y el Caribe: Su visibilidad en SciELO, RedALyC y SCOPUS. Revista Interamericana de Bibliotecología, [S.l.], v. 34, n. 2, p. 187-199, 2011.
MILLER, A.; TAYLOR, S.; BEDEIAN, A. Publish or perish: Academic life as management faculty live it. Career Development International, [S.I.], v. 16, n. 5, p. 422445, 2011.

NASCIMENTO, L. Modelos Capes de avaliação: Quais as consequências para o triênio 2010-2012?

Administração: Ensino e Pesquisa, [S.l.], v. 11, n. 4, p. 579-600, 2010.

NASCIMENTO, S.; BEUREN, I. Redes sociais na produção científica dos programas de pós-graduação de ciências contábeis do Brasil. Revista de Administração Contemporânea, [S.l.], v. 15, n. 1, p. 47-66, 2011.

OLIVEIRA, C.; SANTIN, D.; VANZ, S. Revista Em Questão: Uma análise da sua trajetória a partir dos critérios Qualis: 2003-2012. Em Questão, [S.l.], v. 21, n. 1, p. 8-25, 2015.

PACKER, A. El SciELO para nuestras revistas científicas. Interciência, [S.1.], v. 27, n. 6, p. 274, 2002.

PACKER, A. Os periódicos brasileiros e a comunicação da pesquisa nacional. Revista USP, [S.l.], n. 89, p. 26-61, 2011.

PACKER et al. SciELO - 15 years of open access: an analytic study of open access and scholarly communication. Paris: UNESCO, v.186 p., 2014.

ROCHA-E-SILVA, M. O novo Qualis, que não tem nada a ver com a ciência do Brasil: Carta aberta ao presidente da CAPES. Clinics, [S.1.], v. 64, p. 721-724, 2009.

RODRIGUES, R.; OLIVEIRA, A. Periódicos científicos na America Latina: Títulos em acesso aberto indexados no ISI e SCOPUS. Perspectivas em Ciência da Informação, [S.1.], v. 17, n. 4, p. 77-99, 2012.

ROSA, A. "Nós e os índices": Um outro olhar sobre a pressão institucional por publicação. Revista de Administração de Empresas, [S.l.], v. 48, n. 4, p. 108114, 2008.

SAMPAIO, F. Pesquisa em cirurgia, pós-graduação stricto sensu e sistema de avaliação da Capes. Revista do

Colégio Brasileiro de Cirurgióes, [S.1.], v. 33, n. 5, p. 343, 2006. 
SANTOS, S.; NORONHA, D. Periódicos brasileiros de Ciências Sociais e Humanidades indexados na base SciELO: Características formais. Perspectivas em

Ciência da Informação, [S.l.], v. 18, n. 2, p. 2-16, 2013.

SEGLEN, P. Why the impact factor of journals should not be used for evaluating research. British Medical Journal, [S.l.], v. 314, n. 7.079, p. 498-513, 1997.

SHUGAN, S. Journal rankings: Save the outlets for your research. Marketing Science, [S.I.], v. 22, n. 4, p. 437 441, 2013.

SOUSA, C.; MACEDO, E. Avaliação da pesquisa em Educação e indicadores de produção bibliográfica: Um relato sobre o Qualis periódicos. Revista de Educação Pública, [S.l.], v. 18, n. 37, p. 255-272, 2009.

STAHL, M.; LEAP, L.; WEI, Z. Publication in leading management journals as a measure of institutional research productivity. Academy of Management Journal, [S.l.], v. 31, n. 3, p. 707-720, 1988.

STREHL, L. O fator de impacto do ISI e a avaliação da produção científica: aspectos conceituais e metodológicos.

Ciência da Informação, [S.l.], v. 34, n. 1, p. 19-27, 2005.
STUMPF, I. Passado e futuro das revistas científicas.

Ciência da Informação, [S.l.], v. 25, n. 3, p. 1-6, 1996.

TOURINHO, M.; PALHA, M. A Capes, a universidade e alienação gestada na pós-graduação. Cadernos EBAPE, [S.l.], v. 12, n. 2, p. 270-283, 2014.

TREIN, E.; RODRIGUES, J. O mal-estar na Academia: Produtivismo, o conhecimento-mercadoria. Revista Brasileira de Educação, [S.1.], v. 16, n. 48, p. 769-792, 2011.

TRUEGER et al. The Altmetric score: A new measure for article-level dissemination and impact. Annals of Emergency Medicine, [S.I.], p. 1-5, 2015.

VELASCO et al. La utilización de los indicadores bibliométricos para evaluar la actividad investigadora. Aula Abierta, [S.l.], v. 40, n. 2, p. 75-84, 2012. 